\title{
TOPOLOGICAL ALGEBRAS IN WHICH ALL MAXIMAL TWO-SIDED IDEALS ARE CLOSED
}

\author{
MATI ABEL \\ University of Tartu, 2 Liivi St., 50409 Tartu, Estonia \\ E-mail: abel@ut.ee \\ KRZYSZTOF JAROSZ \\ Southern Illinois University, Edwardsville, IL 62026, U.S.A. \\ E-mail: kjarosz@siue.edu \\ http://www.siue.edu/ ${ }^{\sim}$ kjarosz
}

\begin{abstract}
We characterize unital topological algebras in which all maximal two-sided ideals are closed.
\end{abstract}

1. Introduction. In a unital Banach algebra all maximal two-sided ideals are obviously closed. The problem of characterizing other classes of topological algebras with the same property has been investigated by a number of authors. For example for a complex unital commutative Fréchet algebra that property is equivalent to being a $Q$-algebra ([5], Proposition 17); for a complex unital commutative complete locally $m$-convex algebra for which the set of all non-zero continuous linear multiplicative functionals is compact in the Gelfand topology our property is equivalent to any proper finitely generated ideal being non-dense ([17], Proposition 2). However there are also non- $Q$-algebras with that property $([9]$, p. 81$)$. In this note we provide a characterization of that property in terms of compactness of the structure space and properties of finitely generated ideals. We also show that every proper finitely generated two-sided ideal in a complex unital locally $m$-pseudoconvex Fréchet algebra is contained in a closed maximal two-sided ideal of $A$ if $A$ is topologically almost commutative or $\operatorname{comm} A \neq A$.

Unless otherwise stated the result is valid in both the real and the complex case.

2000 Mathematics Subject Classification: Primary 46H05; Secondary 46H20.

Key words and phrases: topological algebras, Fréchet algebras, locally $m$-pseudoconvex algebras, simplicial algebras, $Q$-algebras.

Research partially supported by a grant from the National Research Council.

The paper is in final form and no version of it will be published elsewhere. 
2. Definitions and notation. By a topological algebra we mean an algebra $A$ that is also a topological vector space such that the multiplication operation is separately continuous.

We call a topological algebra $A$ locally pseudoconvex if it has a base of neighborhoods of zero consisting of balanced and pseudoconvex sets, that is of sets $U$ for which $\mu U \subset U$, whenever $|\mu| \leq 1$, and $U+U \subset \lambda U$ for some $\lambda \geq 2$. In such an algebra the topology can be defined by a family $\left\{p_{\lambda}: \lambda \in \Lambda\right\}$ of $k_{\lambda}$-homogeneous seminorms with $k_{\lambda} \in(0,1]$, where a seminorm $p$ is called $k$-homogeneous if $p(\mu a)=|\mu|^{k} p(a)$ for any $a \in A$ and any scalar $\mu$ ([13], p. 4). If $A$ is also metrizable then one can select a countable family $\left\{p_{\lambda}: \lambda \in \Lambda\right\}$ of $k_{\lambda}$-homogeneous seminorms. A locally pseudoconvex algebra $A$ is locally $m$-pseudoconvex if every seminorm $p_{\lambda}$ in the family $\left\{p_{\lambda}: \lambda \in \Lambda\right\}$ is submultiplicative, is locally convex, if $k_{\lambda}=1$ for each $\lambda \in \Lambda$, and $k$-normed if the topology of $A$ can be define by a single $k$-homogeneous norm. A metrizable and complete algebra is called a Fréchet algebra.

For a unital ring $A, M(A)$ denotes the set of all maximal two-sided ideals in $A$. The space $M(A)$ is equipped with the $h k$-topology: $S \subset M(A)$ is closed if $S=H(K(S))$, where $K(S)$ is the intersection of all ideals in $S$ and $H(I)=\{M \in M(A): I \subset M\}$ for any two-sided ideal $I$ of $A$. If $A$ is also equipped with a compatible topology we consider a subset $m(A)$ of $M(A)$ consisting of closed ideals, we use small letters $h$ and $k$ to indicate operations $H$ and $K$ restricted to $m(A)$.

A unital topological algebra $A$ is called simplicial (with respect to two-sided ideals) or normal in the sense of Michael ([11], p. 68) if every closed two-sided ideal of $A$ is contained in a closed maximal two-sided ideal of $A$. For an algebra $A$ let $\operatorname{rad} A$ denote the topological radical ([3], p. 27) of $A,\left\langle a_{1}, \ldots, a_{n}\right\rangle$ the two-sided ideal of $A$ generated by $a_{1}, \ldots, a_{n} \in A$, and $\operatorname{comm} A$ the commutator ideal of $A$, that is, the closure of the two-sided ideal of $A$ generated by the set $\{a b-b a: a, b \in A\} . A$ is an topologically almost commutative algebra if $A / \operatorname{rad} A$ is commutative.

3. Topological algebras in which all maximal two-sided ideals are closed. The next theorem characterizes topological algebras with closed maximal ideals.

TheOrem 1. Let $A$ be a unital topological algebra. Then $M(A)=m(A)$ if and only if

(i) every proper finitely generated two-sided ideal of $A$ is contained in a closed maximal two-sided ideal of $A$, and

(ii) $m(A)$ is compact in the hk-topology.

To prove the theorem we will need the compactness of $M(A)$, that fact is well known but typically it is presented only for certain classes of algebras or topological algebras (e.g. [8], p. 301, [12], p. 84, or [16]). Below we show that the standard arguments work for an arbitrary unital ring.

Proposition 1. Let $A$ be a ring with a unit e. Then the space $M(A)$ is compact in the $h k$-topology.

Proof. Assume $\mathcal{F}=\left\{F_{j}: j \in J\right\}$ is a nonempty family of $h k$-closed subsets of $M(A)$ with $\bigcap_{j \in J} F_{j}=\varnothing$. We have 


$$
\begin{aligned}
\varnothing & =\bigcap_{j \in J} F_{j}=\bigcap_{j \in J} H K\left(F_{j}\right)=\bigcap_{j \in J}\left\{M \in M(A): K\left(F_{j}\right) \subset M\right\} \\
& =\left\{M \in M(A): K\left(F_{j}\right) \subset M \text { for all } j\right\}=\left\{M \in M(A): \bigcup_{j \in J} K\left(F_{j}\right) \subset M\right\}
\end{aligned}
$$

so the two-sided ideal generated by $\bigcup_{j \in J} K\left(F_{j}\right)$ is equal to the entire ring $A$. Hence there are elements $a_{1}, \ldots, a_{n}$ (not necessarily all different) of $\bigcup_{j \in J} K\left(F_{j}\right)$ and $b_{1}, \ldots, b_{n}$, $b_{1}^{\prime}, \ldots, b_{n}^{\prime}$ in $A$ with $\sum_{k=1}^{n} b_{k}^{\prime} a_{k} b_{k}=e$; let $F_{1}, \ldots, F_{n} \in \mathcal{F}$ be such that $a_{k} \in K\left(F_{k}\right)$ for $k=1,2, \ldots, n$. It follows that the two-sided ideal generated by $\bigcup_{k=1}^{n} K\left(F_{k}\right)$ is equal to the entire $\operatorname{ring} A$ and consequently we can reverse the last sequence of equalities:

$$
\begin{aligned}
\varnothing & =\left\{M \in M(A): \bigcup_{k=1}^{n} K\left(F_{k}\right) \subset M\right\}=\left\{M \in M(A): K\left(F_{k}\right) \subset M \text { for } k=1, \ldots, n\right\} \\
& =\bigcap_{k=1}^{n}\left\{M \in M(A): K\left(F_{k}\right) \subset M\right\}=\bigcap_{k=1}^{n} H K\left(F_{k}\right)=\bigcap_{k=1}^{n} F_{k},
\end{aligned}
$$

which shows that $M(A)$ is compact.

Proof of Theorem 1. Assume $M(A)=m(A)$. In a unital algebra any proper two-sided ideal is contained in a maximal ideal. Hence, if $M(A)=m(A)$ any proper two-sided ideal is contained in a closed maximal two-sided ideal. Since $M(A)$ is compact in the $h k$-topology so is $m(A)$.

Assume now $A$ is a unital topological algebra which satisfies the conditions of the Theorem and let $M_{0} \in M(A)$. Put

$$
Z(a) \stackrel{d f}{=}\{M \in m(A): a \in M\}, \quad \text { for } a \in M_{0} .
$$

Since for any $a_{1}, \ldots, a_{n} \in M_{0}$ we have $I \stackrel{d f}{=}\left\langle a_{1}, \ldots, a_{n}\right\rangle \subset M_{0} \neq A$, by the first condition there is an ideal $M \in m(A)$ with $\left\{a_{1}, \ldots, a_{n}\right\} \subset I \subset M$. Hence $M \in \bigcap_{k=1}^{n} Z\left(a_{k}\right)$, so $\left\{Z(a): a \in M_{0}\right\}$ is a collection of $h k$-closed subsets of $m(A)$ having the finite intersection property. By (ii) there is a closed maximal two-sided ideal $M_{1} \in \bigcap_{a \in M_{0}} Z(a)$. The ideal $M_{1}$ contains all elements of $M_{0}$ so $M_{0} \subset M_{1}$, however $M_{0}$ is maximal so $M_{0}=M_{1} \in m(A)$.

Corollary 1. Let $A$ be a unital simplicial (with respect to two-sided ideals) algebra. If

(a) no proper finitely generated two-sided ideal of $A$ is dense in $A$, and

(b) $m(A)$ is compact in the hk-topology,

then $M(A)=m(A)$.

Proof. Let $I$ be a proper finitely generated two-sided ideal in $A$. Since $\operatorname{cl}_{A}(I)$ is a closed two-sided ideal in a simplicial algebra $A$, by (a) there is $M \in m(A)$ with $\operatorname{cl}_{A}(I) \subset M$. Hence $A$ has the property (i) of Theorem 1 and consequently $M(A)=m(A)$.

EXAMPLE 1. We construct a commutative unital normed algebra $A$ such that both $M(A)$ and $m(A)$ are compact but different. Let $A$ be the space of all $C^{\infty}$ (real or complex valued) functions $f$ defined on the real line $\mathbb{R}$ such that both restrictions $f_{\mid(-\infty, 0]}$ and $f_{\mid[1, \infty)}$ are 
polynomials. We equip $A$ with a submultiplicative norm:

$$
\|f\|=\sup \{|f(t)|: 0 \leq t \leq 1\} .
$$

Notice that $\|\cdot\|$ is indeed a norm rather then just a seminorm since if $f=0$ on the unit segment, then all the derivatives of $f$ at the points 0 and 1 are equal to zero and consequently $f=0$ on the entire line.

Let $I$ be a maximal proper ideal of $A$. Assume there is $f \in I$ which does not vanish on $[0,1]$. If $p_{n}$ is a sequence of polynomials convergent uniformly to $1 / f$ on $[0,1]$ then the sequence $f p_{n}$ converges in $A$ to the unit of that algebra, so $I$ is not closed. On the other hand if all of the functions from $I$ vanish somewhere on $[0,1]$ then the sup norm closure of $I$ in the Banach algebra $C[0,1]$ is a proper ideal so $I \subset\left\{f: f\left(x_{I}\right)=0\right\}$ for some $x_{I}$ in $[0,1]$. Hence $m(A)$ can be identified with the unit segment.

We show that the $h k$-topology on $[0,1]$, denoted by $\tau_{h k}$, coincides with the usual topology $\tau_{s t d}$ of that segment. Clearly any $h k$-closed set is closed in the standard topology so $\tau_{h k} \subset \tau_{s t d}$. On the other hand, since for any $0 \leq a<b \leq 1$ there is a function $f_{0} \in A$ such that $f_{0}(t) \neq 0$ exactly when $a<t<b$, we have $h k([0,1] \backslash(a, b))=[0,1] \backslash(a, b)$ so all of the segments $(a, b)$ are $h k$-open; as such segments form a basis of $\tau_{\text {std }}$ we get $\tau_{\text {std }} \subset \tau_{h k}$.

Hence $m(A)=[0,1]$ while $M(A)$ is much bigger containing as a proper subset all of $\mathbb{R}$.

4. Properties of locally $m$-pseudoconvex Fréchet algebras. The next theorem shows that the first condition considered in the previous section is valid for a large class of topological algebras.

TheOREM 2. Let $A$ be a complex unital locally $m$-pseudoconvex Fréchet algebra. If $\operatorname{comm} A \neq A$ or $A$ is topologically almost commutative then every proper finitely generated two-sided ideal in $A$ is contained in a closed maximal two-sided ideal of $A$.

The idea of proof comes from [6]; we first will need the following lemma ([8], p. 233).

Lemma 1. Let $A$ and $B$ be topological algebras with unit elements $e_{A}$ and $e_{B}$, respectively and let $h$ be a homomorphism from $A$ onto a dense subset of $B$ with $h\left(e_{A}\right)=e_{B}$. Assume $a_{1}, \ldots, a_{n}, c_{1}, \ldots, c_{n} \in A$ and $d_{1}, \ldots, d_{n} \in B$ are such that

$$
\sum_{v=1}^{n} c_{v} a_{v}=e_{A} \text { and } \sum_{v=1}^{n} d_{v} h\left(a_{v}\right)=e_{B} .
$$

Then for any neighborhood $O$ of zero in $B$ there exist $b_{1}, \ldots, b_{n} \in A$ such that

$$
\sum_{v=1}^{n} b_{v} a_{v}=e_{A} \text { and } h\left(b_{v}\right) \in d_{v}+O, \text { for } v=1, \ldots, n \text {. }
$$

Proof of Theorem 2. Assume $A$ is a complex locally $m$-pseudoconvex Fréchet algebra with a unit element $e_{A}$ and the topology given by a family $\left\{p_{n}: n \in \mathbb{N}\right\}$ of $k_{n}$-homogeneous submultiplicative seminorms, with $k_{n} \in(0,1]$, for $n \in \mathbb{N}$. We may also assume ([7], Proposition 4.6.1), that

$$
p_{n}(a)^{\frac{1}{k_{n}}} \leqslant p_{n+1}(a)^{\frac{1}{k_{n+1}}}, \quad \text { for } n \in \mathbb{N}, a \in A, k_{n+1} \leqslant k_{n} .
$$


Put

$$
B \stackrel{d f}{=}\left\{\begin{array}{l}
A / \operatorname{comm} A \text { if } \operatorname{comm} A \neq A \\
A / \operatorname{rad} A \quad \text { if } A \text { is topologically almost commutative, }
\end{array}\right.
$$

let $\kappa: A \rightarrow B$ be the canonical homomorphism, and let

$$
q_{n}(b) \stackrel{d f}{=} \inf \left\{p_{n}(a): \kappa(a)=b, a \in A\right\}, \quad n \in \mathbb{N}, b \in B .
$$

Since $\operatorname{comm} A$ and $\operatorname{rad} A$ are closed two-sided ideals, $B$ is a commutative complex locally $m$-pseudoconvex Fréchet algebra with a unit element $e_{B}=\kappa\left(e_{A}\right)$ and the topology given by the family $\left\{q_{n}: n \in \mathbb{N}\right\}$ of $k_{n}$-homogeneous submultiplicative seminorms. Furthermore by (1)

$$
q_{n}(b)^{\frac{1}{k_{n}}} \leqslant q_{n+1}(b)^{\frac{1}{k_{n+1}}}, \quad \text { for } n \in \mathbb{N}, b \in B, k_{n+1} \leqslant k_{n} .
$$

Next for $n \in \mathbb{N}$ let $\pi_{n}$ be the canonical homomorphism of $B$ onto $B_{n} \stackrel{d f}{=} B / \operatorname{ker} q_{n}$ and let $r_{n}$ be the quotient $k_{n}$-homogeneous norm on $B_{n}$. Let $\widetilde{B}_{n}$ be the completion of $B_{n}$, let $\tilde{r}_{n}$ be the extension of $r_{n}$ to a $k_{n}$-homogeneous norm on $\widetilde{B}_{n}$, and denote by $\mu_{n}: B \rightarrow \widetilde{B}_{n}$ the composition of $\pi_{n}$ with the embedding into $\widetilde{B}_{n}$.

For $m \leqslant n$ we have $B_{n} \subset B_{m}$ and by (2)

$$
r_{m}\left(\pi_{m}(b)\right)^{\frac{1}{k_{m}}}=q_{m}(b)^{\frac{1}{k_{m}}} \leqslant q_{n}(b)^{\frac{1}{k_{n}}}=r_{n}\left(\pi_{n}(b)\right)^{\frac{1}{k_{n}}}, \quad b \in B, m \leqslant n .
$$

For $n, m \in \mathbb{N}$ with $m \leqslant n$ let

$$
f_{m, n}: B_{n} \rightarrow B_{m}, \quad \text { be defined by } f_{m, n}\left(\pi_{n}(b)\right)=\pi_{m}(b) .
$$

By (3) the homomorphism $f_{m n}$ is a uniformly continuous map from $B_{n}$ onto $B_{m}$ ([7], Theorem 4.3.11) so it can be continuously extended to a homomorphism $\tilde{f}_{m, n}$ from the commutative $k_{n}$-Banach algebra $\widetilde{B}_{n}$ onto a dense subalgebra of $k_{m}$-Banach algebra $\widetilde{B}_{m}$ ([10], Proposition 5, p. 129). We have

$$
\tilde{f}_{l, n}=\tilde{f}_{l, m} \circ \tilde{f}_{m, n}, \text { and } \tilde{r}_{m}\left(\tilde{f}_{m, n}\left(\tilde{b}_{n}\right)\right)^{\frac{1}{k_{m}}} \leqslant \tilde{r}_{n}\left(\tilde{b}_{n}\right)^{\frac{1}{k_{n}}}, \quad \text { for } l \leqslant m \leqslant n, \tilde{b}_{n} \in \widetilde{B}_{n} .
$$

To finish the proof assume $I$ is a finitely generated ideal in $A$ and let $a_{1}, \ldots, a_{s} \in A$ be such that $I=\left\langle a_{1}, \ldots, a_{s}\right\rangle$. Suppose that

$$
\left\langle\mu_{n}\left(\kappa\left(a_{1}\right)\right), \ldots, \mu_{n}\left(\kappa\left(a_{s}\right)\right)\right\rangle=\tilde{B}_{n}, \quad \text { for all } n \in \mathbb{N},
$$

and let $\tilde{b}_{1}^{n}, \ldots, \tilde{b}_{s}^{n} \in \tilde{B}_{n}$ be such that

$$
\sum_{v=1}^{s} \tilde{b}_{v}^{n} \mu_{n}\left(\kappa\left(a_{v}\right)\right)=\mu_{n}\left(\kappa\left(e_{A}\right)\right)=e_{\widetilde{B}_{n}} .
$$

Put $\tilde{d}_{v}^{n-1}=\tilde{f}_{n-1, n}\left(\tilde{b}_{v}^{n}\right)$. We have

$$
\sum_{v=1}^{s} \tilde{d}_{v}^{n-1} \mu_{n-1}\left(\kappa\left(a_{v}\right)\right)=\mu_{n-1}\left(\kappa\left(e_{A}\right)\right)=e_{\widetilde{B}_{n-1}} .
$$

Hence by Lemma 1 , with $A=\tilde{B}_{n}, B=\tilde{B}_{n-1}, O=\left\{x \in \tilde{B}_{n-1}: \tilde{r}_{n-1}(x)<2^{-n}\right\}$, and $h=\tilde{f}_{n-1, n}$ there are $\tilde{d}_{1}^{n}, \ldots, \tilde{d}_{s}^{n} \in \tilde{B}_{n}$ such that

$$
\sum_{v=1}^{s} \tilde{d}_{v}^{n} \mu_{n}\left(\kappa\left(a_{v}\right)\right)=e_{\widetilde{B}_{n}}
$$


and

$$
\tilde{r}_{n-1}\left(\tilde{f}_{n-1, n}\left(\tilde{d}_{v}^{n}\right)-\tilde{d}_{v}^{n-1}\right)<2^{-n}, \quad n \in \mathbb{N}, v=1, \ldots, s .
$$

By (4) for $m \leqslant n-1$ and $v=1, \ldots, s$

$$
\begin{aligned}
\tilde{r}_{m}\left(\tilde{f}_{m, n}\left(\tilde{d}_{v}^{n}\right)-\tilde{f}_{m, n-1}\left(\tilde{d}_{v}^{n-1}\right)\right) & =\tilde{r}_{m}\left(\tilde{f}_{m, n-1}\left(\tilde{f}_{n-1, n}\left(\tilde{d}_{v}^{n}\right)\right)-\tilde{f}_{m, n-1}\left(\tilde{d}_{v}^{n-1}\right)\right) \\
& =\tilde{r}_{m}\left(\tilde{f}_{m, n-1}\left(\tilde{f}_{n-1, n}\left(\tilde{d}_{v}^{n}\right)-\tilde{d}_{v}^{n-1}\right)\right) \\
& \leqslant\left(\tilde{r}_{n-1}\left(\tilde{f}_{n-1, n}\left(\tilde{d}_{v}^{n}\right)-\tilde{d}_{v}^{n-1}\right)\right)^{\frac{k_{m}}{k_{n-1}}} \leqslant\left(\frac{1}{2^{n}}\right)^{\frac{k_{m}}{k_{n-1}}}
\end{aligned}
$$

therefore, since $k_{l} \leqslant k_{n}$ for $l \geqslant n$, for any $n \leqslant p<q$ we get

$$
\tilde{r}_{n}\left(\tilde{f}_{n, q}\left(\tilde{d}_{v}^{q}\right)-\tilde{f}_{n, p}\left(\tilde{d}_{v}^{p}\right)\right) \leqslant \sum_{t=p+1}^{q} \tilde{r}_{n}\left(\tilde{f}_{n, t}\left(\tilde{d}_{v}^{t}\right)-\tilde{f}_{n, t-1}\left(\tilde{d}_{v}^{t-1}\right)\right)<\sum_{t=p+1}^{q}\left(\frac{1}{2^{t}}\right)^{\frac{k_{n}}{k_{t-1}}} \leqslant \sum_{t=p+1}^{q}\left(\frac{1}{2}\right)^{t} .
$$

Hence, as $\sum_{t=0}^{\infty}\left(\frac{1}{2}\right)^{t}$ is convergent, $\left(\tilde{f}_{n, n+l}\left(\tilde{d}_{v}^{n+l}\right)\right)_{l \in \mathbb{N}}$ is a Cauchy sequence in $\tilde{B}_{n}$, for any $n \in \mathbb{N}$ and $v=1, \ldots, s ;$ as $\tilde{B}_{n}$ are complete we may put

$$
\lim _{l \rightarrow \infty} \tilde{f}_{n, n+l}\left(\tilde{d}_{v}^{n+l}\right) \stackrel{d f}{=} \tilde{e}_{v}^{n} \in \tilde{B}_{n} .
$$

By (4) $\left(\tilde{e}_{v}^{n}\right)_{n \in \mathbb{N}} \in \varliminf_{\longleftarrow}\left\{\tilde{B}_{n}, \tilde{f}_{m, n}, \mathbb{N}\right\}$ for each $v=1, \ldots, s$. Since

$$
B \ni b \mapsto\left(\mu_{n}(b)\right) \in \varliminf_{\longleftarrow}\left\{\tilde{B}_{n}, \tilde{f}_{m, n}, \mathbb{N}\right\}
$$

is a surjective topological isomorphism ([2], pp. 18-22, or [7], Theorem 4.5.3) there are elements $e_{1}, \ldots, e_{s} \in A$ such that

$$
\mu_{n}\left(\kappa\left(e_{v}\right)\right)=\tilde{e}_{v}^{n}=\lim _{l \rightarrow \infty} \tilde{f}_{n, n+l}\left(\tilde{d}_{v}^{n+l}\right), \quad \text { for all } v \text { and } n .
$$

Therefore by (5) and (6) for each $n \in \mathbb{N}$ we get

$$
\begin{aligned}
p_{n}\left(\sum_{v=1}^{s} e_{v} a_{v}-e_{A}\right) & =\tilde{r}_{n}\left(\mu_{n}\left(\kappa\left(\sum_{v=1}^{s} e_{v} a_{v}-e_{A}\right)\right)\right) \\
& =\tilde{r}_{n}\left(\sum_{v=1}^{s} \mu_{n}\left(\kappa\left(e_{v}\right)\right) \mu_{n}\left(\kappa\left(a_{v}\right)\right)-\mu_{n}\left(\kappa\left(e_{A}\right)\right)\right) \\
& =\tilde{r}_{n}\left(\sum_{v=1}^{s} \lim _{l \rightarrow \infty} \tilde{f}_{n, n+l}\left(\tilde{d}_{v}^{n+l}\right) \mu_{n}\left(\kappa\left(a_{v}\right)\right)-\mu_{n}\left(\kappa\left(e_{A}\right)\right)\right) \\
& =\lim _{l \rightarrow \infty} \tilde{r}_{n}\left(\tilde{f}_{n, n+l}\left(\sum_{v=1}^{s} \tilde{d}_{v}^{n+l} \mu_{n+l}\left(\kappa\left(a_{v}\right)\right)-\mu_{n+l}\left(\kappa\left(e_{A}\right)\right)\right)\right)=0,
\end{aligned}
$$

hence $I=A$. So for every proper finitely generated ideal $I$ of $A$ there is $n_{0} \in \mathbb{N}$ such that $\mu_{n_{0}}(\kappa(I)) \neq \tilde{B}_{n_{0}}$. Because $\mu_{n_{0}}(\kappa(I))$ is an ideal in a complex unital commutative $k_{n_{0}}$-Banach algebra $\tilde{B}_{n_{0}}$ there is a nontrivial continuous multiplicative linear functional $\varphi_{0}$ on $\tilde{B}_{n_{0}}$ such that $\mu_{n_{0}}(\kappa(I)) \subset \operatorname{ker} \varphi_{0}$ ([14], Proposition 4.3, or [15], Theorem 4.1). Let $\phi=\varphi_{0} \circ \mu_{n_{0}} \circ \kappa$. Then $\phi$ is a nontrivial continuous linear multiplicative functional on $A$, $\operatorname{ker} \phi$ is a closed maximal two-sided ideal in $A$ and $I \subset \operatorname{ker} \phi$. 
Corollary 2. Assume $A$ is a complex unital locally m-pseudoconvex Fréchet algebra that is topologically almost commutative, or such that $\operatorname{comm} A \neq A$. Then $M(A)=m(A)$ if and only if $m(A)$ is compact in the hk-topology.

5. Simplicial algebras with compact topological strong structure space. In this section we discuss the second condition listed in Theorem 1. The following proposition generalizes Corollary 3.9 of ([1]); notice that every commutative $Q$-algebra satisfies the condition (7) below.

Proposition 2. Let $A$ be a unital simplicial algebra. If

$$
\operatorname{cl}_{A}\left(\bigcup_{M \in m(A)} M\right) \subset \bigcup_{M \in M(A)} M,
$$

then $m(A)$ is compact in the hk-topology.

Proof. Suppose $m(A)$ is not compact and let $\left(F_{\gamma}\right)_{\gamma \in \Gamma}$ be a family of $h k$-closed subsets of $m(A)$ with the finite intersection property and such that $\bigcap_{\gamma \in \Gamma} F_{\gamma}=\varnothing$. Let $J$ be the two-sided ideal in $A$ generated by $\left\{k\left(F_{\gamma}\right): \gamma \in \Gamma\right\}$. Since $A$ is simplicial, if $\operatorname{cl}_{A}(J) \neq A$ then $\operatorname{cl}_{A}(J) \subset M$ for some $M \in m(A)$. As $k\left(F_{\gamma}\right) \subset \operatorname{cl}_{A}(J) \subset M$ for every $\gamma \in \Gamma$, then $M \in h\left(k\left(F_{\gamma}\right)\right)=F_{\gamma}$ for each $\gamma \in \Gamma$, which contradicts our assumption. Hence $\operatorname{cl}(J)=A$.

Fix $a \in A$ and a neighborhood of zero $O$ in $A$. Let $O^{\prime}$ be a neighborhood of zero in $A$ such that $O^{\prime} a \subset O$. Since $A$ is unital $\left(e_{A}+O^{\prime}\right) \cap J \neq \varnothing$ and we can find $o \in O^{\prime}, n \in \mathbb{N}$, $\gamma_{1}, \ldots, \gamma_{n} \in \Gamma$ and $a_{\gamma_{k}} \in k\left(F_{\gamma_{k}}\right)$ such that

$$
e_{A}+o=\sum_{k=1}^{n} a_{\gamma_{k}} \in \sum_{k=1}^{n} k\left(F_{\gamma_{k}}\right)
$$

Let $M_{o} \in \bigcap_{k=1}^{n} F_{\gamma_{k}}$. Since $M_{o} \in F_{\gamma_{k}}=h\left(k\left(F_{\gamma_{k}}\right)\right)$ so $k\left(F_{\gamma_{k}}\right) \subset M_{o}$, and we get

$$
e_{A}+o \in M_{o} \subset \bigcup_{M \in m(A)} M .
$$

Hence

$$
\left(e_{A}+O\right) \bigcap\left(\bigcup_{M \in m(A)} M\right) \neq \varnothing, \quad \text { for any neighborhood } O \text { of zero in } A .
$$

Consequently

$$
e_{A} \in \operatorname{cl}_{A}\left(\bigcup_{M \in m(A)} M\right) \subset \bigcup_{M \in M(A)} M
$$

which is impossible.

Corollary 3. Assume $A$ is a complex unital locally m-pseudoconvex Fréchet algebra that is topologically almost commutative, or such that $\operatorname{comm} A \neq A$. If $A$ satisfies the condition (7) then $M(A)=m(A)$.

Proposition 3. A commutative unital simplicial topological algebra $A$ is a $Q$-algebra if and only if it satisfies (7) and $M(A)=m(A)$. 
Proof. If $A$ is a commutative $Q$-algebra then $M(A)=m(A)$ and (7) holds because $A \backslash \operatorname{Inv} A$ is equal to the union of all ideals from $M(A)$. If $A$ is a commutative unital topological algebra and $M(A)=m(A)$ then the union of all ideals from $m(A)$ and the union of all ideals from $M(A)$ coincides; by (7) the last union is closed so $A$ is a $Q$-algebra.

It is clear that every $Q$-algebra is simplicial. The next result characterizes those simplicial algebras which are $Q$-algebras.

Theorem 3. Let $A$ be a commutative unital simplicial algebra. Then $A$ is a $Q$-algebra if and only if A satisfies (7) and condition (a) of Corollary 1.

Proof. If $A$ is a commutative $Q$-algebra then $M(A)=m(A)$ and $\bigcup_{M \in M(A)} M=A \backslash \operatorname{Inv} A$ is closed in $A$.

Assume $A$ is a commutative unital simplicial algebra and $I$ is a proper finitely generated ideal in $A$. If $A$ satisfies the condition (a) of Corollary 1 then there is an ideal $M \in m(A)$ such that $I \subset M$. By Theorem 1 and Proposition 2 we have $M(A)=m(A)$. Hence, by (7) $\operatorname{Inv} A=A \backslash \bigcup_{M \in M(A)} M$ is open in $A$ and consequently $A$ is a $Q$-algebra.

Corollary 4. Let $A$ be a commutative unital complex locally m-pseudoconvex Fréchet algebra. Then $A$ is a $Q$-algebra if and only if $A$ satisfies (7).

Proof. By Theorem 4.2 of [4] algebra $A$ is simplicial and by Theorem 2 it satisfies condition (a) of Corollary 1. Hence the result follows from Theorem 3.

\section{References}

[1] Mart Abel, Structure of Gelfand-Mazur algebras, Dissertationes Math. Univ. Tartuensis 31, Tartu Univ. Press, Tartu, 2003.

[2] Mati Abel, Projective limits of topological algebras, Tartu. Ülik. Toimetised 836 (1989), 3-27 (in Russian).

[3] Mati Abel, Descriptions of the topological radical in topological algebras, in: General topological algebras (Tartu, 1999), Math. Stud. (Tartu) 1, Est. Math. Soc., Tartu, 2001, 25-31.

[4] Mati Abel, Inductive limits of Gelfand-Mazur algebras, Int. J. Pure Appl. Math. 16 (2004), 363-378.

[5] M. Akkar et C. Nacir, Continuité automatique dans les limites inductives localement comvexes et Q-algèbres de Fréchet, Ann. Sci. Math. Québec 19 (1995), 85-96.

[6] R. F. Arens, Dense inverse limit rings, Michigan Math. J. 5 (1958), 169-182.

[7] V. K. Balachandran, Topological Algebras, North-Holland Math. Stud. 185, Elsevier, Amsterdam, 2000.

[8] E. Beckenstein, L. Narici and Ch. Suffel, Topological Algebras. North-Holland Math. Studies 24, North-Holland, Amsterdam-New York-Oxford, 1977.

[9] R. Choukri, Sur certaines questions concernant les Q-algèbres, Extracta Math. 16 (2001), 79-82.

[10] J. Horváth, Topological Vector Spaces and Distributions, Vol. I, Addison-Wesley, Reading, Mass., 1966.

[11] E. A. Michael, Locally multiplicatively-convex topological algebras. Mem. Amer. Math. Soc., 1952.

[12] C. E. Rickart, General Theory of Banach Algebras, D. van Nostrand, Princeton, 1960. 
[13] L. Waelbroeck, Topological Vector Spaces and Algebras, Lecture Notes in Math. 230, Springer-Verlag, Berlin, 1973.

[14] W. Żelazko, Metrical generalizations of Banach algebras, Dissertationes Math. 47 (1965).

[15] W. Żelazko, Selected Topics in Topological Algebras, Lect. Notes Ser. 31, Aarhus Univ., 1971.

[16] W. Żelazko, Banach Algebras, Elsevier, Amsterdam and PWN-Polish Scientific Publishers, Warsaw, 1973.

[17] W. Żelazko, On maximal ideals in commutative $m$-convex algebras, Studia Math. 57 (1976), 291-298. 